\title{
A 26-year-old man with substance abuse, agitation, and pneumomediastinum
}

\begin{abstract}
A 25year old male with a history of substance abuse (cocaine, marijuana, and alcohol), anxiety, and psychosis was brought to the emergency department (ED) by law enforcement officers. The officers had responded to a call from his wife during a domestic dispute. On their arrival he was found to be disrobed and incoherent and was threatening his wife with a knife. He was subdued and brought to the ED in handcuffs for evaluation of altered mental status and agitation.
\end{abstract}

Volume I Issue 2 - 2014

\author{
Scott Aberegg, Bryce R Erickson, Mark \\ Cowan \\ Jordan Valley Medical Center, USA
}

Correspondence: Scott Aberegg, Jordan Valley Medical Center, USA, Tel 8016642180,Email bryceroger@yahoo.com

Received:September 12, 2014 | Published: September 22, 2014
Abbreviations: ECD, electronic control devices; ED, emergency department; ExDS, excited delirium syndrome; CEWs, conducted electrical weapons

\section{Introduction}

The patient had no other past medical history. He had recently been discharged from an inpatient psychiatry unit for psychosis and was prescribed risperidone, but had not been taking it. He had surgery for a jaw fracture remotely. Because of agitated delirium, psychotic behavior and combativeness, he was physically restrained in the ED and haloperidol was given in repeat doses.

On physical examination, he was a febrile with a pulse of 136, BP $135 / 68$, respiratory rate $20, \mathrm{SpO} 296 \%$ on 3 liters nasal cannula. He was agitated and having visual hallucinations. His voice was muffled. He had abrasions on his hip and shoulder and marked crepitus throughout the neck and upper chest. Trachea was midline. There was a small skin defect overlying the larynx consistent with minor trauma. Lung fields

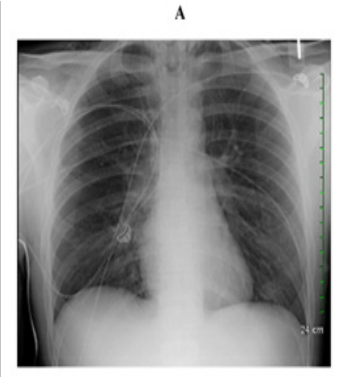

C

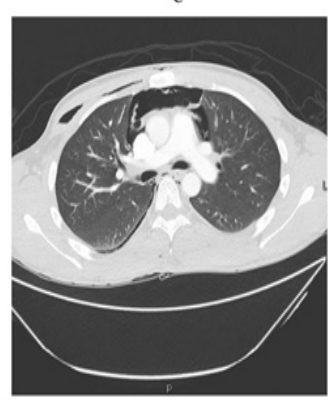

Figure I Chest X-ray and representative CT images of the chest. were clear and abdomen was benign. One examiner thought that there may have been a Haman's crunch, but because of extensive crepitus over the thorax this could not be confirmed. Neurological examination was grossly non-focal.

Laboratory results were remarkable a WBC count of 21,000, HCO3- of 5.7, lactate level of $5.0 \mathrm{mmol} / \mathrm{L}$, a CK level of 21,000 , a creatinine level of 1.8 , and a urine toxicology screen positive for amphetamines and cocaine.

\section{Radiographic findings}

Chest X-ray and representative CT images of the chest are shown (Figure 1).

What are the diagnosis and etiology?

Diagnosis: Pneumomediastinum, pneumopericardium and pneumothorax caused by TASER injury of the larynx and Valsalva maneuver in a patient with Excited Delirium Syndrome (ExDS)
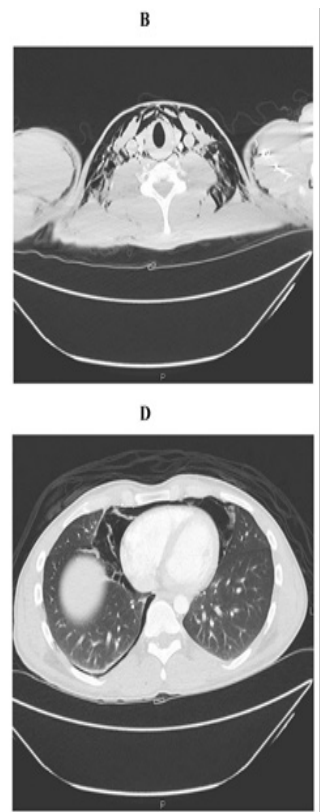


\section{Discussion}

Law enforcement agencies have increasingly adopted non-lethal tactics to subdue subjects during apprehension and arrest, and the use of Electronic Control Devices (ECDs; also referred to Conducted Electrical Weapons, CEWs) such as the TASER has increased dramatically in the last decade. This device delivers a low current, high voltage electric shock via two barbed electrode darts (Figure 2) that are fired into the skin of the subject at distances of up to 35 feet. The electrical discharge causes both intense pain and the involuntary contraction of skeletal muscles, temporarily incapacitating the subject and allowing him to be subdued with less risk of injury to arresting officers and, in theory, the subject himself. Several categories of adverse medical effects of ECD use have been reported in the literature and must be considered in subjects in police custody undergoing medical evaluation. In addition, because widespread use of ECDs is a recent development, novel mechanisms of injury must be considered.

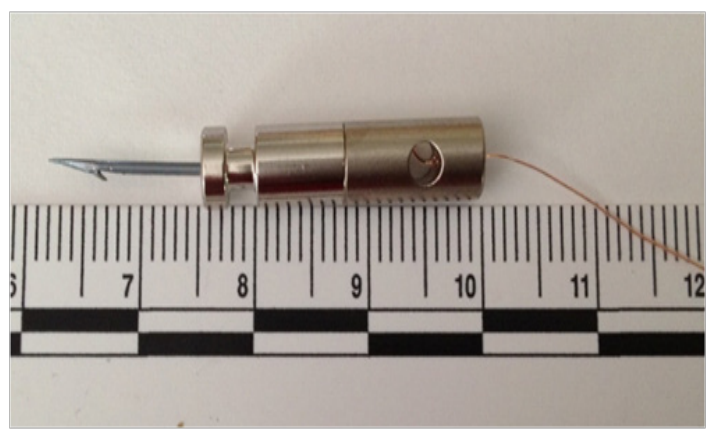

Figure 2 TASER dart.

Much attention has been focused on fatalities after ECD exposure and the possible role of induction of cardiac arrhythmias by ECD use during apprehension and arrest. While experimental studies and extensive anecdotal evidence (approximately half of TASER exposures have involved volunteers during TASER training sessions) have generally failed to document adverse cardiac effects of ECD exposure in healthy volunteers under controlled conditions, the majority of ECD exposures in the field have involved young male subjects who were under the influence of drugs and alcohol. ${ }^{1}$ The presence of these substances confounds attempts to determine the independent contribution of the ECD discharge to adverse outcomes. With one notable recent exception, retrospective investigations of fatalities after field exposure to ECDs have failed to conclude that ECDs cause malignant arrhythmias and death..$^{1-3}$ ECD use has been determined in most studies to be a potential or contributory factor in a minority of deaths, but evidence of bias on the basis of study funding has also emerged. ${ }^{4}$ In any case, fatalities after ECD use are rare, and the mechanism of death in these instances is a subject of ongoing inquiry.

Excited Delirium Syndrome (ExDS) is a clinical entity characterized by altered mental status, adrenergic hyper activation, "superhuman strength", hyperthermia, acidosis, aggressiveness, high tolerance to pain and violent resistance to restraint, often in patients with psychiatric disturbances and acute or chronic drug abuse especially that involving stimulants. Like our patient, they may also have recently stopped antipsychotic medications and are often inappropriately clothed. These patients have a documented increased risk of sudden death, ${ }^{5}$ and this association further confounds attempts to determine the causality of fatalities in patients who have both ExDS and ECD exposure. Treatment of ExDS is supportive and includes benzodiazepines and major tranquilizers such as haloperidol.

The marked tonic muscle contraction that occurs during ECD discharge can lead to rhabdomyolysis, transient lactic acidosis, and even vertebral compression fractures. Loss of postural control can cause falls, with resultant trauma including head injuries. Penetrating trauma from ECD darts is a rare but diverse category of serious injury from ECD use. Puncture of the globe of the eye and the cranium with cerebral injury have been reported in addition to minor injuries from penetration of extremities and non-vital organs. ${ }^{1}$ Pharyngeal and laryngeal perforations as well as pneumothorax have been described in case reports.

In the absence of symptoms in an awake and alert patient, laboratory testing, EKGs, telemetry monitoring, and prolonged ED or inpatient observation is not recommended after ECD exposure. ${ }^{6}$ The initial evaluation and treatment of our patient in the emergency department appropriately focused on his excited delirium, and supportive care with fluids and oxygen was administered. Interviews with arresting officers revealed that our patient was thought to have been struck with the TASER darts in the abdomen and upper chest, but based on our physical examination we determined that one dart had penetrated his larynx. We reason that the tissue planes of his larynx and neck were violated by the dart, allowing air to dissect through tissue planes continuous with the mediastinal and pleural spaces. During the TASER discharge (or afterwards while struggling with law enforcement officers), forceful exhalation against a closed glottis (Valsalva maneuver) forced air between the injured tissue planes in the neck and resulted in Pneumomediastinum, pneumothorax, pneumopericardium, subcutaneous air, and muffled voice from air around the larynx. Our case highlights the need to consider novel mechanisms of injury in patients with ECD exposure.

The ease with which minor injury of a vulnerable tissue plane followed by Valsalva maneuver can lead to dissection of air is not widely recognized but was demonstrated by a case series in this journal in 2001. ${ }^{7}$ Lopez-Pelaez et al., ${ }^{7}$ reported subcutaneous emphysema, Pneumomediastinum, and pneumothorax of unclear cause in a series off our prisoners from a single penitentiary in Spain. It was subsequently discovered that they had intentionally punctured tissues in the oral cavity with needles and fish bones and induced air dissection via Valsalva maneuver, with suspected escape intent.

Our patient was monitored in the ICU overnight and his lactic acidosis resolved. His Pneumomediastinum, pneumothorax, and rhabdomyolysis resolved over several days with supportive case and his mental status also improved with reinstitution of his chronic medications and dissipation of the effects of illicit drugs.

\section{Clinical pearls}

The vast majority of exposures to ECDs do not result in significant injury and testing and monitoring are not warranted in uncomplicated cases; nonetheless, patients exposed to ECDs are at risk for several categories of injury, and novel forms of injury must be considered depending on the clinical scenario. Patients with drug and alcohol intoxication as well as ExDS appear to be at increased risk of injury and death in association with ECD exposure. Trauma and falls from loss of postural control are significant causes of injury from ECD exposure; lactic acidosis and rhabdomyolysis from forceful muscular contractions are usually self-limited. Pneumomediastinum, 
pneumothorax, and subcutaneous air are possible after ECD exposure due to penetrating trauma, Valsalva maneuver, or some combination thereof. Relatively minor injury to the tissues of the oropharynx and neck can lead to dissection of air, especially if accompanied by Valsalva maneuver.

\section{Acknowledgements}

None.

\section{Conflict of interest}

The author declares no conflict of interest.

\section{References}

1. Pasquier M, Carron PN, Vallotton L, et al. Electronic control device exposure: a review of morbidity and mortality. Ann Emerg Med. 2011;58(2):178-188.

2. Zipes DP. Sudden cardiac arrest and death following application of shocks from a TASER electronic control device. Circulation 2012;125(20):2417-2422.
3. Swerdlow CD, Fishbein MC, Chaman L, et al. Presenting rhythm in sudden deaths temporally proximate to discharge of TASER conducted electrical weapons. Acad Emerg Med. 2009;16(8):726-739.

4. Azadani PN, Tseng ZH, Ermakov S, et al. Funding source and author affiliation in TASER research are strongly associated with a conclusion of device safety. Am Heart J. 2011;162(3):533-537.

5. Vilke GM, DeBard ML, Chan TC, et al. Excited delirium syndrome (ExDS): defining based on a review of the literature. $J$ Emerg Med. 2012;43(5):897-905.

6. Vilke GM, Bozeman WP, Chan TC. Emergency department evaluation after conducted energy weapon use: review of the literature for the clinician. J Emerg Med. 2011;40(5):598-604.

7. Lopez-Pelaez MF, Roldan J, Mateo S. Cervical emphysema, pneumomediastinum, and pneumo thorax following self-induced oral injury: report of four cases and review of the literature. Chest. 2001;120(1):306-309. 